\title{
Economic value of clinical decision support allied to direct data feedback to clinicians:
}

\section{blood usage in haematology}

Nicholas Swart ${ }^{1}$, Stephen Morris ${ }^{1,2}$, Michael F Murphy ${ }^{3,4}$

${ }^{1}$ Department of Applied Health Research, University College London, London

${ }^{2}$ Department of Public Health and Primary Care, University of Cambridge

${ }^{3}$ NIHR Biomedical Research Centre Blood Theme, Oxford University Hospitals and University of Oxford

${ }^{4}$ NHS Blood \& Transplant

Corresponding author details:

Mr Nicholas Swart

Department of Applied Health Research

University College London

1-19 Torrington Place, London WC1E 7HB

n.swart@ucl.ac.uk

Tel: 02031083348

Mob: 07919314346

The authors declare that they have no conflicts of interest regarding the submitted article.

Running title: Cost impact of transfusion decision support 
Keywords: haematology; blood transfusion; health economics; clinical decision support system; interrupted time series analysis;

\section{Abstract}

Background and Objectives Responding to national and local pressures to reduce the amount of blood transfused, the haematology department of Oxford University Hospitals (OUH), UK implemented an electronic blood-ordering system with clinical decision support. This intervention targeted junior doctors, giving regular feedback on their transfusion practices with respect to clinical guidelines.

Methods We evaluated the incremental costs of the intervention using Interrupted Time Series methods to compare red blood cell and platelet usage before and after the intervention was implemented. Difference-in-differences analysis was used to control for external factors that would affect the use of blood products over time. Reductions in blood usage were balanced against intervention costs.

Results The base case analysis showed an average cost-saving to the department of $£ 89,304$ annually as a result of the intervention. Scenario analyses suggested that the savings may have been greater still, had the increasing trend in blood use prior to the intervention continued in the absence of the intervention.

Conclusion An electronic blood-ordering system with clinical decision support can reduce blood transfusions and associated health care costs. Focusing on improving junior doctors' transfusion practice is expected to have a knock-on benefit in terms of dissemination of good transfusion practice both within their own department and others as they continue their training. 


\section{Introduction}

Blood transfusions have been identified as one of the most over-used therapies both in the United States and in England through the 'Choosing Wisely' initiatives, which support evidence-based care to minimize the harms of over treatment; both initiatives made recommendations about good transfusion practice.[1,2] Variation in transfusion rates for commonly used indications in developed countries persists despite the publication of numerous clinical practice guidelines which might have been expected to standardise practice.[3]

Patient Blood Management (PBM) is an increasingly used international term to describe an evidencebased, multidisciplinary approach to optimising the care of patients who might need transfusion.[4] It encompasses measures to avoid transfusion such as anaemia management without transfusion, cell salvage and the use of anti-fibrinolytic drugs to reduce bleeding as well as restrictive transfusion. It ensures that patients receive the optimal treatment, and that avoidable, inappropriate use of blood and blood components is reduced. Restrictive transfusion practice, an example of this approach, is blood transfusion therapy given only when the potential benefits are deemed to outweigh potential risks, and in which there is a goal of minimising the use of blood.[5] While the risks of transfusion are known, its benefits to patients are less certain outside the immediate management of major haemorrhage. A 2016 Cochrane meta-analysis of 31 trials in over 12,000 patients compared restrictive transfusion to liberal transfusion strategies, and found no difference in 30 day mortality or any of the other outcomes assessed including cardiac events, myocardial infarction, stroke, thromboembolism, and infection.[6] The implications are that restrictive as opposed to liberal transfusion practices do not cause harm in key outcomes while reducing the quantities of blood transfused and number of times patients receive transfusions. Recent studies suggest that the use of electronic blood ordering with clinical decision support systems (CDSS) may provide an effective way of implementing restrictive transfusion practice and for providing timely data on blood usage so that clinicians and clinical teams can compare their practice with others.[7-11] These studies report how CDSS can reduce non- 
evidence-based transfusions and increase compliance with restrictive transfusion policies, but their effectiveness improves when coupled with education of clinicians, and when combined may even improve clinical outcomes.

Blood utilisation in hospitals is undergoing renewed scrutiny not only in relation to clinical patient outcomes, but also because of the potential for significant cost-savings by hospitals: not only in the acquisition cost of the units of blood, but also in laboratory testing, storage of blood, administering blood and monitoring patients. It is hoped that an emphasis on reducing costs, and particularly unnecessary costs, will support the evidence on restrictive transfusion practice and other PBM measures to accelerate these into routine transfusion practice.

This study is based on an intervention in a large academic medical centre, which aimed to provide further understanding about how best to configure a CDSS for blood transfusion to provide feedback of data to optimise clinicians' behaviour, specifically targeting junior doctors. This observational study aimed to evaluate whether a CDSS with regular feedback meetings successfully reduced blood transfusion. Analysing the cost-implications of this system was the key objective of the study.

\section{Methods}

\section{Study Design and data}

We conducted a retrospective analysis of the number and cost of blood products used by Oxford University Hospitals (OUH) haematology department, compared with the rest of OUH. The OUH is a system covering 2 acute hospitals, a specialist orthopaedic centre and a regional hospital providing non-emergency services. During the period covered by this intervention the haematology department consisted of 25 haematology beds, supported by a general intensive care unit and an oncology/haematology triage department for acute admissions. Monthly usage data on units of red blood cells (RBC) and units of platelets were obtained from January 2012 to December 2016. Data 
were also collected on the number of blood transfusion episodes, and the number of patient bed days.

\section{The CDSS and feedback intervention}

The implementation of the CDSS and feedback of data to the clinical team in the OUH haematology department started in May 2014, providing a clear differentiation between the Before and After intervention period. The CDSS was developed by the Oxford team within the hospitals' Cerner Millennium ${ }^{\circledast}$ electronic patient records (EPR) system. When ordering blood products using the CDSS, haematologists enter relevant clinical characteristics, and are alerted when a combination of the most recent blood count and clinical information fall outside of locally agreed parameters based on national guidelines for blood transfusion.[12] These parameters differed depending on blood product being requested (for more information see Butler et al. 2015) [10] but, as an example, the acceptable indications for RBC were:

- Massive bleeding with blood pressure instability;

- $\quad \mathrm{HB} \leq 7 \mathrm{~g} / \mathrm{dL}$ in a stable ICU patient;

- $\quad \mathrm{HB} \leq 8.0 \mathrm{~g} / \mathrm{dL}$ in a non-ICU patient with signs/symptoms of anaemia;

- $\quad H B \leq 10 \mathrm{~g} / \mathrm{dL}$ with acute cardiac ischemia;

- Surgical blood loss anticipated;

- $\quad$ Other (free text).

Data on the number and types of transfusions collected by the system were developed into monthly reports on clinician's transfusion practices by a Transfusion Practitioner (TP), part-time at 0.8 Full Time Equivalent, and fed back to junior haematology doctors in one-hour monthly meetings. In these meetings, all the transfusions given outside guidelines in the previous month are reviewed and education provided about good transfusion practice. The meetings are led by a senior haematology Consultant and the Specialist Registrar (SpR) in haematology on rotation through transfusion medicine. Twelve junior doctors rotate through the haematology ward each year, eight of whom are in Core Training stage 1 (CT1) and 4 of whom are SpRs in haematology stage 3. Each monthly 
meeting is attended by the $3 \mathrm{CT} 1 \mathrm{~s}$ and the ward $\mathrm{SpR}$. The doctor who ordered the transfusion explains their decision to transfuse providing information about clinical and/or laboratory factors which might justify the transfusion. There is open discussion to promote sharing of experience and the education aspect of the meeting. It should be noted that the Transfusion Practitioner provides training to clinical staff throughout $\mathrm{OUH}$, not only in haematology, on the use of the electronic blood-ordering and the other electronic transfusions systems. The TP was also involved in the design of the CDSS. It may be possible to reduce the amount of time needed by the TP to produce monthly reports. In the base case analysis we costed the TP at 0.8 FTE as this was appropriate to the project at the time these data were recorded, and because it is not clear whether less TP time would produce lower quality reports and have a lower impact on the reduction of volume of blood transfusions. We explored in Scenario 4 of the sensitivity analysis the effects of reducing TP time to 0.4 FTE, as the intervention cost was a significant proportion of the net cost savings.

\section{Measuring costs}

We evaluated the average incremental monthly cost of blood product usage before the intervention start date with the costs of blood product usage after the intervention was introduced. Differencein-Differences (DiD) analysis was then conducted comparing the differences in costs of blood product usage before and after the introduction of the intervention in the haematology department, and the difference in the rest of OUH (excluding haematology) over the same time period.

Unit cost data on blood products were sourced from the National Health Service Blood and Transplant (NHSBT) Price List for 2017/18.[13] The acquisition cost of a unit of standard red blood cells (RBC) was $£ 124.46$, and for a unit of platelets was $£ 178.19$. The cost of administering a unit of blood used was $£ 40.18$. We estimated this by taking the difference between our unit price of blood and the value given for the "total cost of transfusing a subsequent unit of blood" in the NICE guidelines on management of blood transfusion costing statement [14], and inflating it to 2017/18. Therefore the unit costs for RBC and platelets was $f 164.64$ and $£ 218.37$ respectively. The time 
horizon for the analyses was 60 months, based on data collected for 28 months before May 2014, and 32 months after and including May 2014. The study took an NHS perspective. The cost components included were units of RBC and of platelets, and the running cost of the intervention.

\section{Cost of the intervention}

The CDSS intervention relies upon having a hospital-wide Electronic Patient Record (EPR) system in place. The CDSS is a module added onto that system by EPR staff with input from haematology staff at the design phase. There are no ongoing costs or overheads born by the haematology department for the continued running of the EPR or the CDSS module in particular. The Transfusion Practitioner and a senior haematology consultant were responsible for designing the module, and a member of the EPR staff was responsible for implementing it on the EPR system. after consultation with project staff we have estimated the development cost of the CDSS module to be the sum of approximately 25 hours of EPR staff time (Band $6-£ 14.74$ per hour), 20 hours of TP time (Band $7-£ 17.60$ per hour) and 5 hours of senior consultant time ( $£ 44.27$ per hour); a total of $£ 941.90$ for developing and implementing the CDSS. This cost has not been included in our analysis as it is not an ongoing cost. The intervention does, however, incur ongoing- costs in the form of 30-minute training sessions for new junior doctors on how to use the system (at a cost of $£ 115$ of staff time per annum), and onehour monthly meetings attended by $3 \mathrm{CT} 1 \mathrm{~s}, 1 \mathrm{SpR}$, a senior haematology consultant and the TP. Collecting and analysis the data collected by the CDSS is also not an insignificant cost in terms of TP time. The total annual running costs of the intervention was therefore estimated at $£ 30,899$ (the majority of which is the Transfusion Practitioner's salary). Table 2 shows the relevant staff costs for the intervention.

\section{Statistical analysis}

To model blood product usage over time we used linear regression to predict usage trends, and interrupted time series (ITS) methodology to differentiate between an intervention group and a 
control group. The predicted outputs of these regression models were used in the economic evaluation and difference-in-differences analysis.

First, we adopted a before-after design using ITS methods [15] to generate predicted values for use in the cost analysis. This involved using a series of monthly data points for volume of blood products used in the haematology department to establish a trend, which is 'interrupted' by the intervention. A new trend was established from the start of the intervention, which was then compared with the pre-intervention trend as well as hypothetical 'counter-factual' scenarios such as if the intervention had not taken place and the 'pre-intervention' trend had continued uninterrupted.

Second, linear piecewise regression was used to compare the 'Before' period with the 'After' period in the haematology department as well as the rest of $\mathrm{OUH}$, and to estimate the predicted trends of each period for use in the cost analysis. We then compared the slopes of the predicted trends to see if there was a significant difference between the Before slope and the After slope for haematology, and separately for the rest of OUH.

The cost of blood usage for the month of May 2014 was included in the After period only to avoid double-counting. We did not 'force' the trends of each period to join, permitting a drop in predicted blood usage between April and May 2014. This reflected the relatively sudden impact of the intervention 'going live' in May 2014. We explore the effect of allowing this drop in the sensitivity analysis.

We then conducted difference-in-differences (DiD) analysis [16] to estimate the impact of the intervention, controlling for any changes in the volume of blood product usage in the rest of OUH excluding the haematology department, to account for underlying trends in blood usage that may have affected haematology. This involved estimating the change in the volume of blood products used by the rest of OUH between the Before and After periods, and comparing that with the change in the volume of blood products used by the haematology department. The analysis controlled for patient bed days in order to account for the vastly different size of the rest of OUH compared to the 
haematology department. The average monthly number of bed days for haematology were 731 and 733 in the Before and After periods, respectively; for the rest of OUH the average monthly numbers were 39,266 and 42,055 bed days, respectively. As such, monthly bed usage was relatively consistent in haematology between the Before and After periods. Although there is a slight increase in OUH bed days, this was very unlikely to have been caused by this intervention in haematology, given the difference in size between haematology and OUH, the consistency of haematology bed days, and the overall downward trend in blood use in OUH. In order to convert our DiD results back into a useful unit of measure when calculating the average monthly use of blood products, we multiplied the DiD outputs by 733.156 which is the exact figure for the average number of bed days in haematology in the After period. The ITS and DiD analysis were both conducted using Stata15. [17]

\section{Cost Analysis}

Using the outputs from the regression models, we calculated the average monthly use of blood products for the Before and After periods for haematology and for the rest of OUH. The difference in average monthly blood usage and associated cost can be considered the incremental savings between the Before and After periods. The cost analysis was conducted using Microsoft Excel 2016.

\section{Sensitivity analyses}

Deterministic sensitivity analysis was used to examine a number of potential scenarios that may have occurred in the absence of the intervention. Four counterfactual scenarios for the ITS analysis were explored:

- Scenario 1: if the Before trend had continued uninterrupted from January 2012 to December 2016

- Scenario 2: if the Before trend stopped at May 2014 and blood product usage stayed constant from the level in May 2014 until December 2016 (i.e. if the intervention halted the increasing use of RBC at May 2014, but did not start reducing the use of RBC post-May 2014) 
- Scenario 3: if there was no level change allowed by the regression model (i.e. if there was no sudden drop in blood product usage in May 2014 to coincide with the implementation of the intervention), but the trend in the After period was the same as in the base case.

- Scenario 4: if the cost of the TP was reduced to 0.4 FTE instead of the original 0.8 FTE.

\section{National Budget Impact}

We calculated an estimate of the total savings nationally that could be achieved if every acute hospital in England had a haematology department that reduced their volume of blood product use by the equivalent of that seen in this study.

\section{Results}

Interrupted-Time Series Analysis of blood usage data

Reference to the raw data on blood usage in the Supplementary Materials suggests that the trend lines predicted by the linear piecewise regression are appropriate, and that after May 2014 there was a sharp change in actual blood usage levels. Supplementary Figures $1 \& 2$ show the raw data and trend lines for RBC and Platelets, respectively for haematology, and Supplementary Figures 3 \& 4 show the same for the rest of $\mathrm{OUH}$.

Table 1 describes the predicted coefficients from the ITS regressions for haematology and for the rest of the OUH. For haematology, the Before period showed a statistically significant increase in the use of $\mathrm{RBC}$ and platelets per month $(1.3,95 \% \mathrm{Cl} 0.2$ to 2.3 ; and $2.1,95 \% \mathrm{Cl} 0.8$ to 3.4 respectively). The After period showed a statistically significant decrease in the use of RBC per month $(-1.6,95 \% \mathrm{Cl}$ -2.5 to -0.7$)$ but no statistically significant decrease in the use of platelets per month $(-0.03,95 \% \mathrm{Cl}$ 1.1 to 1.0$)$.

For both RBC and platelets the difference between the pre- and post-intervention use of blood was statistically significant $(p<.05)$. See Figures 1 and 2 , which show the predicted trend lines for blood use for the Before and After periods for haematology, for RBC and platelets, respectively, based on 
the coefficients in Table 1. These figures also show the trend lines for the sensitivity analysis scenarios.

For the rest of $\mathrm{OUH}$, the Before period showed a decrease in monthly RBC that was not statistically significant $(-3.1,95 \% \mathrm{Cl}-10.0$ to 3.9$)$, but showed a statistically significant increase in the use of platelets per month $(2.7,95 \% \mathrm{Cl} 1.0$ to 4.4$)$. The After period shows a statistically significant decrease in use of RBC per month $(-9.6,05 \% \mathrm{Cl}-15.3$ to -4.0$)$, and showed an increase in monthly platelets use that was not statistically significant $(0.7,95 \% \mathrm{Cl}-0.7$ to 2.2$)$. For both $\mathrm{RBC}$ and platelets there was no significant difference between the pre- and post-intervention use of blood. Graphs depicting the actual volume of blood transfused for haematology, and regression trend lines for the rest of $\mathrm{OUH}$, are shown in Supplementary Materials 1 and 2.

Cost analysis (base case)

Using the outputs from the regression models we estimated the total volume of blood used and the average monthly volume of blood used by haematology and the rest of OUH for the Before and After periods (Table 3). The total number of units of blood product used were generated by the regression model based on data from haematology and the rest of OUH.

For RBCs there was a difference of 47.5 units $(£ 7,822)$ between the average monthly blood use levels of the Before and After periods. This indicates an average annual cost-savings of $£ 93,865$ (i.e. an annual reduction of 570 units of RBC).

For platelets there was a difference of 10.1 units $(£ 2,195)$ between the average monthly blood use levels of the Before and After periods. This indicates an average annual cost-savings of $f 26,337$ (121 units of platelets). Therefore, the total cost-savings (less the cost of the intervention itself) was £89,304 per year. 


\section{Accuracy of the regression models outputs}

We saw only a small degree of difference between the predicted outputs of the regression and the actual volume of blood product usage for haematology. The model over-predicted RBC use by $1.1 \%$ in the Before period, and under-predicted RBC use by $1.3 \%$ in the After period. The model underpredicted platelets use by $0.4 \%$ in the Before period, and over-predicted platelets use by $0.6 \%$ in the After period. This meant a very slight bias for the intervention in the RBC analysis, and against the intervention in the platelets analysis.

\section{Sensitivity analyses}

In order to estimate the bias introduced by the over- and under-estimation of the regression model, we conducted a cost analysis using the actual values for blood usage. In terms of RBC usage, there was an average annual cost-savings of $£ 87,037$ ( 529 units of RBC). In terms of platelets there was an average annual cost-savings of $£ 29,433$ (135 units of platelets). This meant a total cost-savings of $f 85,571$ when considering the cost of the intervention.

We compared the four scenarios against the volume of blood product usage in the After period predicted by the regression model above $(4,056$ units of RBC, costing $£ 667,851$; and 3,418 units of platelets costing $f 746,421$ ) and subtracting the cost of the intervention (Table 4 and Figures 1 and 2).

- Scenario 1 considered the outcome if the Before trends continued beyond May 2014 (i.e. no intervention) and estimated average annual cost-savings of $£ 168,264$ (1022 units) for RBC, and $£ 193,070$ (884 units) for platelets: total savings of $£ 330,435$ per year when considering the cost of the intervention.

- Scenario 2 considered the outcome if the after trend held constant at the level of usage in May 2014 (i.e. no drop in usage after the intervention) and estimated average annual savings of $£ 129,825$ (789 units) for RBC, and $£ 106,925$ (490 units) for platelets: total savings of $£ 205,850$ per year when considering the cost of the intervention. 
- Scenario 3 considered the outcome if the After trend started at the same point the Before trend stopped at in May 2014 (i.e. no sharp drop in blood usage in May 2014) and estimated average annual savings of $£ 80,970$ (492 units) for RBC, and $£ 107,439$ (492 units) for platelets, meaning a total savings of $£ 157,510$ per year when considering the cost of the intervention.

- Scenario 4 considered the outcome if TP time was reduced to $0.4 \mathrm{FTE}$, meaning an intervention cost of $£ 16,464$. This scenario assumes the same RBC and platelet savings as the base case cost analysis (and therefore uses the corresponding trend lines on Figure 1), and estimated a total savings of $£ 103,949$ per year when considering the cost of the intervention.

\section{Difference-in-differences analysis}

The DiD results suggest that haematology saw a significant decrease of 0.061 units of RBC per bed day per month (Table 5). The rest of OUH saw a non-significant decrease of 0.009 units of RBC per bed day per month. This difference-in-differences of -0.051 units of RBC per bed day per month was significantly different from zero. This equates to an average monthly reduction of 37 units of RBC to the haematology department, over and above the changes seen elsewhere in the trust over the same time period. Therefore haematology saw an estimated average annual cost-savings of $£ 73,873$ (i.e. 37 RBC units per month * $£ 164.64$ per RBC unit *12 months) through the reduction of RBC used, when controlling for the change in the rest of $\mathrm{OUH}$.

Regarding platelets, haematology saw a marginally significant decrease of 0.016 units per bed day per month. The rest of OUH saw a non-significant decrease of 0.001 units of platelets per bed day per month. The difference-in-differences of -0.015 was significant at $p<.05$. This equates to an average monthly reduction of 11 units of platelets to the haematology department. Therefore haematology saw an estimated average annual cost-savings of $f 28,694$ (i.e. 11 platelets units per 
month * $f 218.37$ per platelets unit * 12 months) through the reduction of platelets used, when controlling for the change in the rest of $\mathrm{OUH}$.

When considering the combined costs of RBC and platelets, minus the running costs of the intervention itself, this produced an estimated annual cost-saving of $£ 71,669$.

Projected national budget impact

If the cost-savings seen by this intervention were rolled out to all 135 acute non-specialist hospitals in England, then the total cost-savings to the NHS would be $£ 9,675,258$ per year. This assumes that the haematology department or equivalent services for all hospitals are similar to the rest of OUH in their volumes of blood use. This also assumes that the benefits of the intervention would be limited to haematology departments, and there are no 'spill-over' effects of reducing the volume of blood transfused outside of haematology departments, and that as in OUH there would be no set-up costs.

\section{Discussion}

\section{Main findings}

In this study we evaluated the impact of an intervention to reduce the number and associated of cost blood products associated with inappropriate blood transfusions as indicated by the triggering of an alert in the CDSS. Our base case analysis suggested cost saving of $£ 89,304$ each year for the first 3 years after the intervention was introduced. We used counter-factual sensitivity analysis and DiD analyses to explore the real world uncertainty around this result, which produces estimates of cost-savings ranging from $f 71,669$ (DiD) to $£ 330,435$ (Scenario 1 ).

\section{Strengths and limitations}

This study has several strengths. The use of multiple methods of analysis is a strength in that we have tried to 'triangulate' towards as robust an estimate of the economic impact of this intervention as possible. That the base case and the DiD saw similar results is encouraging. Including a DiD 
analysis strengthens our findings by trying to account for system-level initiatives elsewhere in the Trust that would impact the haematology department's use of blood transfusions. The sensitivity analyses are an additional strength: considering other plausible scenarios and comparing this intervention against them helps to contextualise the observed benefits (often difficult for analysis based on observational data). This study is one of the first studies of blood usage to use these methods of analysis, although there is growing interest in using ITS methods [18] to evaluate interventions in the absence of a control group.

This study also has several weaknesses. The absence of a control group is a limitation, despite our attempts to overcome this. We may have underestimated the potential savings by not considering the cost of adverse transfusion events potentially avoided through reducing the number of transfusions. A weakness of the DiD is that haematology patients have a different pattern of blood transfusion needs compared to patients in the rest of $\mathrm{OUH}$, which will have a much more varied mix of patients and their respective transfusion needs (e.g. in surgery and oncology). It is not possible to say whether this means we are under- or over-estimating the potential cost-savings, although it is encouraging that the DiD results were not dissimilar to the ITS results. The training of the junior doctors in haematology may have a ripple effect as they move on to other clinical specialties in the rest of OUH, the benefit of which we have not been able to capture or quantify. Lastly, we do not have any direct evidence from this study to address the concern that fewer transfusions might have led to worse clinical outcomes. However, recent evidence from several randomised controlled trials indicates that strategies for the restrictive use of RBC transfusions are associated with similar or better patient outcomes to liberal RBC transfusion strategies. [6,19-23] In addition, blood transfusion is known to be associated with adverse events, [5] however it was not possible to include these in this analysis. 


\section{Comparison with other studies}

This intervention to reduce the amount of blood transfused is part of both a UK and a worldwide effort to curb the overuse of blood transfusions, thereby saving on costs to the NHS and other health services by reducing 'unnecessary' use of a finite resource. $[5,24]$ Guidance release by NICE (National Institute for Health and Care Excellence) in 2015 recommended methods to reduce blood transfusions with the express benefits of patient safety and saving hospitals money.[12] Using electronic CDSS was an express recommendation for research as part of this guidance. A recent article [25] saw a reduction in RBC transfusions using a CDSS intervention with ITS analysis methods (although theirs was not a health economic analysis). Implementing a clinical decision support system has also seen success elsewhere in the United States with an improvement in patient outcomes as well as reduced use of blood and associated costs; Goodnough reports net savings of $\$ 78,080$ per 100 patient discharges for RBC unit purchases [26].

\section{Conclusion}

The success of this intervention suggests the potential benefits of rolling-out CDSS in haematology departments in other hospitals. Whether the same benefit can be expected by introducing this CDSS to other clinical specialties who transfuse large volumes of blood in the rest of OUH remains to be determined. Previous research has suggested that introducing a CDSS alone is unlikely to achieve significant benefits [27]. As such we suggest that it is the combination of CDSS and the continuing monitoring and feedback with regular meetings, a continuous audit \& feedback loop, that accounts for the success of the intervention. It may also be possible to reduce the cost of the intervention by reducing the amount of TP time spent on training and report writing.

It should also be noted that the cost-savings identified in this study were reliant upon having a preexisting electronic patient records system to build the CDSS on. However, some of the reasons for the success of this intervention are not reliant on having an electronic system, such as the focus on addressing clinical practice with regular meetings and direct data feedback to focus on reducing 
'unnecessary' blood transfusions. The interaction and discussion in these meetings is hugely facilitated by having up-to-date data on transfusion practice: a real-time audit and feedback cycle. Through the active feedback and education provided in these meetings and given the small team and close contact between clinical staff at OUH haematology, it was expected that the effects of the intervention training and scrutiny would rapidly spread to the rest of the team. It was hoped to empower the junior doctors to challenge inappropriate requests by more senior staff, even though senior doctors were not the target of the intervention.

\section{Author Contribution and Acknowledgements}

MM designed and led the study and intervention; NS and SM analysed the data; all authors contributed towards writing and revising the paper. The CDSS was developed by the Oxford team within the hospitals' Cerner electronic patient record (EPR) system. IT is described in more detail in Butler et al. (2015) [10].The data were analysed independently by researchers at University College London. The addition of the CDSS module was considered a service development by OUH and therefore no ethics approval was needed.

\section{References}

[1] American Board of Internal Medicine. Choosing Wisely 2014.

[2] Academy of Medical Royal Colleges. Choosing Wisely recommendations 2014.

[3] Goodnough LT, Levy JH, Murphy MF. Concepts of blood transfusion in adults. Lancet Lond Engl 2013;381:1845-54. https://doi.org/10.1016/S0140-6736(13)60650-9.

[4] Goodnough LT, Shander A. Patient blood management. Anesthesiology 2012;116:1367-76. https://doi.org/10.1097/ALN.0b013e318254d1a3.

[5] Goodnough LT, Murphy MF. Do liberal blood transfusions cause more harm than good? BMJ 2014;349:g6897-g6897. https://doi.org/10.1136/bmj.g6897. 
[6] Carson JL, Stanworth SJ, Roubinian N, Fergusson DA, Triulzi D, Doree C, et al. Transfusion thresholds and other strategies for guiding allogeneic red blood cell transfusion. Cochrane Database Syst Rev 2016;10:CD002042. https://doi.org/10.1002/14651858.CD002042.pub4.

[7] McWilliams B, Triulzi DJ, Waters JH, Alarcon LH, Reddy V, Yazer MH. Trends in RBC ordering and use after implementing adaptive alerts in the electronic computerized physician order entry system. Am J Clin Pathol 2014;141:534-41.

https://doi.org/10.1309/AJCPEN6VHTOECAFI.

[8] Zuckerberg GS, Scott AV, Wasey JO, Wick EC, Pawlik TM, Ness PM, et al. Efficacy of education followed by computerized provider order entry with clinician decision support to reduce red blood cell utilization. Transfusion (Paris) 2015;55:1628-36.

https://doi.org/10.1111/trf.13003.

[9] Tim Goodnough L, Andrew Baker S, Shah N. How I use clinical decision support to improve red blood cell utilization. Transfusion (Paris) 2016;56:2406-11.

https://doi.org/10.1111/trf.13767.

[10] Butler CE, Noel S, Hibbs SP, Miles D, Staves J, Mohaghegh P, et al. Implementation of a clinical decision support system improves compliance with restrictive transfusion policies in hematology patients: CDSS AND BLOOD PRODUCT ORDERING. Transfusion (Paris) 2015;55:1964-71. https://doi.org/10.1111/trf.13075.

[11] Wintermeyer TL, Liu J, Lee KHK, Ness PM, Johnson DJ, Hoffman NA, et al. Interactive dashboards to support a patient blood management program across a multi-institutional healthcare system. Transfusion (Paris) n.d.;56:1480-1. https://doi.org/10.1111/trf.13621.

[12] National Institute for Health and Care Excellent (NICE). Guidance for blood transfusion 2015.

[13] National Health Service Blood Transfusions (NHSBT). Blood and transplant price list 2017/18 2017.

[14] National Institute for Health and Care Excellent (NICE). Costing statement: Blood transfusion; Implementing the NICE guideline on blood transfusion (NG24) 2015. 
[15] Lopez Bernal J, Cummins S, Gasparrini A. Interrupted time series regression for the evaluation of public health interventions: a tutorial. Int J Epidemiol 2016. https://doi.org/10.1093/ije/dyw098.

[16] Card D, Krueger A. Minimum Wages and Employment: A Case Study of the Fast-Food Industry in New Jersey and Pennsylvania. Am Econ Rev 1994;84.

[17] StataCorp. Stata Statistical Software: Release 15. College Station, TX: StataCorp LLC; 2017.

[18] Kontopantelis E, Doran T, Springate DA, Buchan I, Reeves D. Regression based quasiexperimental approach when randomisation is not an option: interrupted time series analysis. BMJ 2015;350:h2750. https://doi.org/10.1136/bmj.h2750.

[19] Carson JL, Terrin ML, Noveck H, Sanders DW, Chaitman BR, Rhoads GG, et al. Liberal or restrictive transfusion in high-risk patients after hip surgery. N Engl J Med 2011;365:245362. https://doi.org/10.1056/NEJMoa1012452.

[20] Hajjar LA, Vincent J-L, Galas FRBG, Nakamura RE, Silva CMP, Santos MH, et al. Transfusion Requirements After Cardiac Surgery: The TRACS Randomized Controlled Trial. JAMA 2010;304:1559-67. https://doi.org/10.1001/jama.2010.1446.

[21] Hébert PC, Martin C, Yetisir E. A Multicenter, Randomized, Controlled Clinical Trial of Transfusion Requirements in Critical Care. N Engl J Med 1999:9.

[22] Lacroix J, Tucci M, Toledano BJ, Meert K. Transfusion Strategies for Patients in Pediatric Intensive Care Units. N Engl J Med 2007:11.

[23] Villanueva C, Colomo A, Bosch A, Concepción M, Hernandez-Gea V, Aracil C, et al. Transfusion Strategies for Acute Upper Gastrointestinal Bleeding. N Engl J Med 2013;368:11-21. https://doi.org/10.1056/NEJMoa1211801.

[24] Hartley S, Foy R, Walwyn REA, Cicero R, Farrin AJ, Francis JJ, et al. The evaluation of enhanced feedback interventions to reduce unnecessary blood transfusions (AFFINITIE): protocol for two linked cluster randomised factorial controlled trials. Implement Sci 2017;12:84. https://doi.org/10.1186/s13012-017-0614-8. 
[25] Kassakian SZ, Yackel TR, Deloughery T, Dorr DA. Clinical Decision Support Reduces Overuse of Red Blood Cell Transfusions: Interrupted Time Series Analysis. Am J Med 2016;129:636.e13-20. https://doi.org/10.1016/j.amjmed.2016.01.024.

[26] Goodnough LT, Maggio P, Hadhazy E, Shieh L, Hernandez-Boussard T, Khari P, et al. Restrictive blood transfusion practices are associated with improved patient outcomes. Transfusion (Paris) 2014;54:2753-9. https://doi.org/10.1111/trf.12723.

[27] Hibbs SP, Noel S, Miles D, Staves J, Murphy MF. The impact of electronic decision support and electronic remote blood issue on transfusion practice. Transfus Med 2014;24:274-9. https://doi.org/10.1111/tme.12149. 


\section{Figures and Tables}

Table 1: linear piecewise regression model results for haematology and the rest of OUH

Haematology

The Rest of OUH

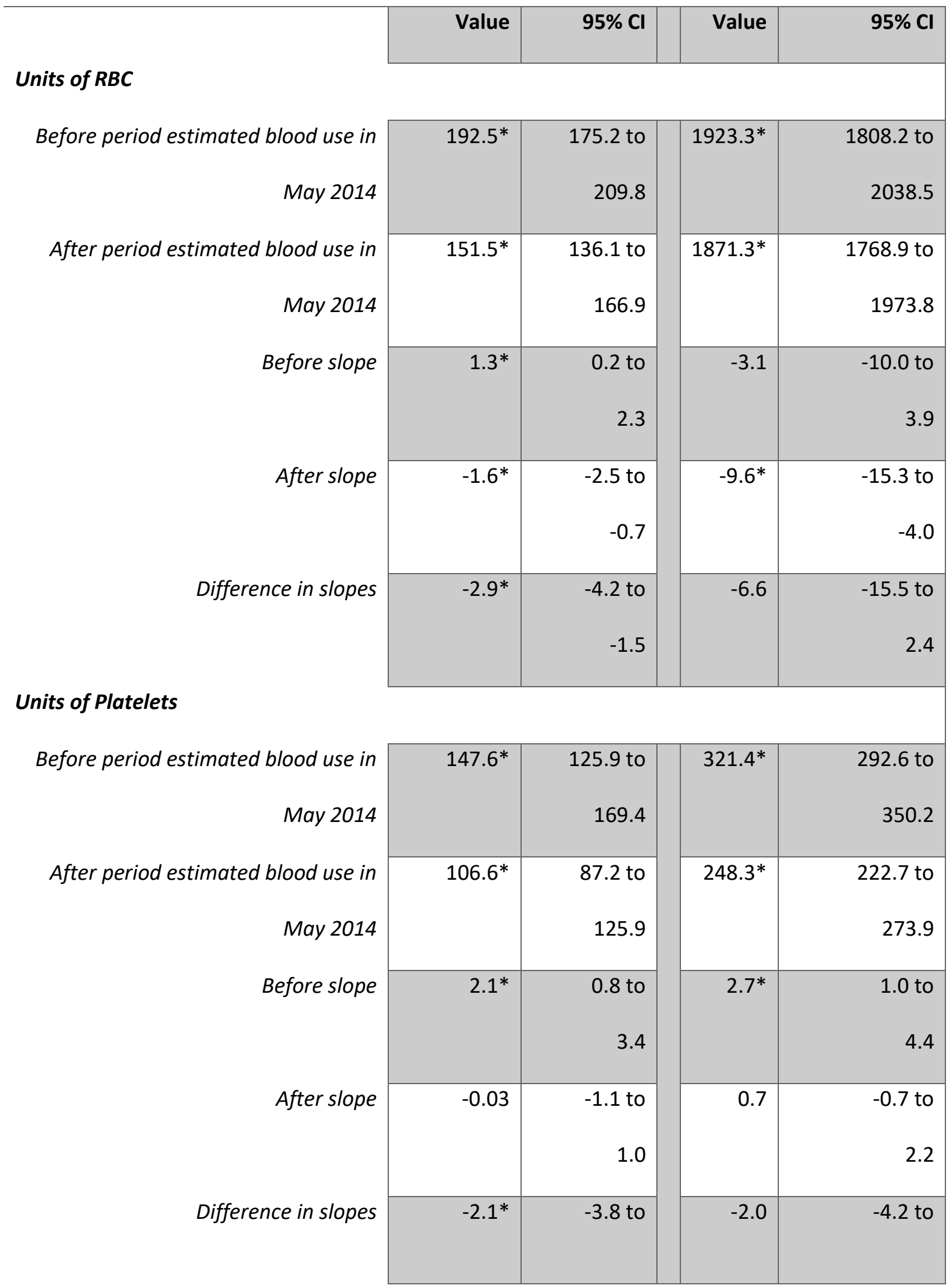


*significant at $p<0.05$

\begin{tabular}{|l|r|r|r|}
\hline-0.4 & & 0.3 \\
& & & \\
\hline
\end{tabular}


Table 2: staff costs

\begin{tabular}{|c|c|c|c|c|}
\hline Role & $\begin{array}{l}\text { Hourly } \\
\text { salary }\end{array}$ & $\begin{array}{l}\text { Additional time spent as } \\
\text { result of intervention }\end{array}$ & $\begin{array}{l}\text { Number } \\
\text { of staff }\end{array}$ & $\begin{array}{r}\text { Intervention cost } \\
\text { (per annum) }\end{array}$ \\
\hline $\begin{array}{l}\text { Junior Doctor } \\
\text { (Core Training 1) }\end{array}$ & $f 18$ & $\begin{array}{r}12 \text { hours (meetings) } \\
0.5 \text { hours system training } \\
\text { (each) }\end{array}$ & 4 & f841 \\
\hline Specialty Registrar & $f 22$ & $\begin{array}{r}12 \text { hours (meetings) } \\
0.5 \text { hours system training } \\
\text { (each) }\end{array}$ & 1 & $£ 267$ \\
\hline Senior Consultant & $f 50$ & $\begin{array}{l}12 \text { hours (meetings) } \\
0.5 \text { hours system training }\end{array}$ & 1 & $£ 597$ \\
\hline Transfusion Practitioner & N/A & New post: $0.8 \mathrm{FTE}$ & 1 & $f 29,290$ \\
\hline
\end{tabular}


Table 3: cost analysis results for haematology using regression outputs

$\begin{array}{crrr}\text { Total Use } & \text { Total Use } & \text { Average monthly } & \text { Average Monthly } \\ \text { Before } & \text { After } & \text { Use Before } & \text { Use After }\end{array}$

Red blood cells

\begin{tabular}{|r|r|r|r|r|}
\hline Number of units of blood & 4879.6 & 4056.4 & 174.3 & 126.8 \\
\hline Cost & $£ 803,389$ & $£ 667,851$ & $£ 28,692$ & $£ 20,870$ \\
\hline
\end{tabular}

Platelets

\begin{tabular}{|r|r|r|r|r|}
\cline { 2 - 5 } Number of units of blood & 3272.3 & 3418.1 & 116.9 & 106.8 \\
\cline { 2 - 5 } Cost & $£ 714,572$ & $£ 746,421$ & $£ 25,520$ & $£ 23,326$ \\
\hline
\end{tabular}


Table 4: sensitivity analysis results

Scenario 1 - If Before trend continued unchecked

Red blood cells

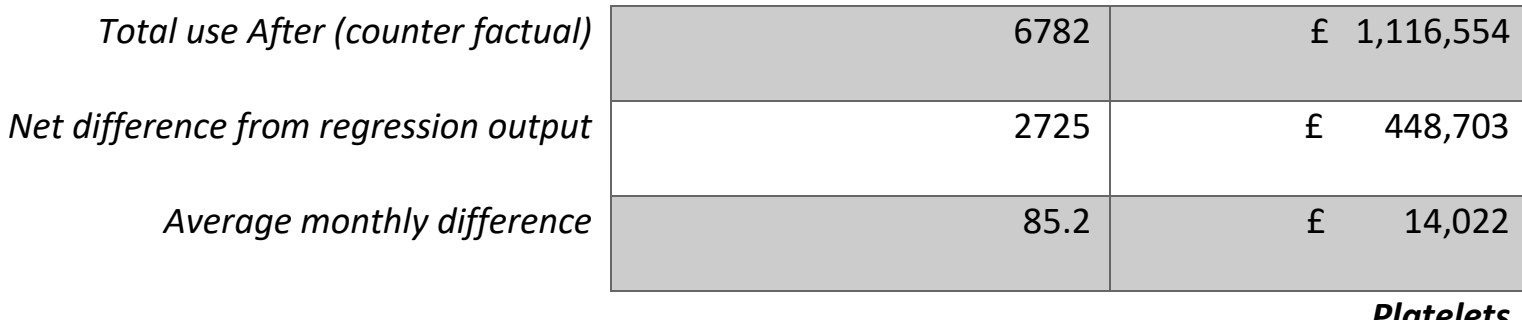

Platelets

\begin{tabular}{r|r|rr|}
\cline { 2 - 4 } Total use After (counter factual) & 5776 & $\mathrm{f} 1,261,274$ \\
\cline { 3 - 4 } Net difference from regression output & 2358 & $\mathrm{f}$ & 514,853 \\
\cline { 2 - 4 } Average monthly difference & 73.7 & $\mathrm{f}$ & 16,089
\end{tabular}

Scenario 2 - If After trend holds constant at May 2014 level without drop in usage at May

2014

Red blood cells

\begin{tabular}{r|r|rr|}
\cline { 2 - 4 } Total use After (counter factual) & 6159 & & $1,014,050$ \\
Net difference from regression output & 2103 & $£$ & 346,199 \\
\cline { 2 - 4 } Average monthly difference & 65.7 & $£$ & 10,819
\end{tabular}

Platelets

\begin{tabular}{r|r|r|}
\cline { 2 - 3 } Total use After (counter factual) & 4724 & $£ 1,031,554$ \\
\cline { 2 - 4 } Net difference from regression output & 1306 & $£ \quad 285,132$ \\
\cline { 2 - 4 } Average monthly difference & 40.8 & $£ \quad 8,910$
\end{tabular}

Scenario 3 - If After trend occurs at the same pace, but without drop in usage at May 2014

\section{Red blood cells}

\begin{tabular}{|c|c|c|c|}
\hline Total use After (counter factual) & 5368 & $f$ & 883,770 \\
\hline Net difference from regression output & 1311 & $f$ & 215,919 \\
\hline
\end{tabular}


Average monthly difference

41.0

f

6,747

Platelets

\begin{tabular}{|c|c|c|c|}
\hline Total use After (counter factual) & 4730 & & 032,925 \\
\hline Net difference from regression output & 1312 & & 286,504 \\
\hline Average monthly difference & 41.0 & $f$ & 8,953 \\
\hline
\end{tabular}


Table 5: Results of the difference-in-differences analysis

Average number of units/bed day SE $p$

\section{$R B C$ units per bed day}

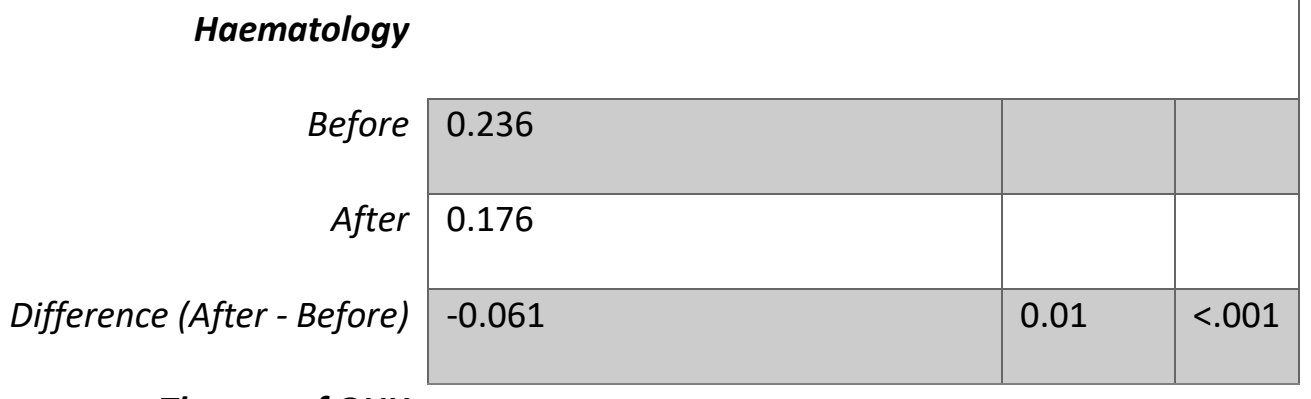

The rest of $\mathrm{OUH}$

\begin{tabular}{r|l|l|l|} 
Before & 0.050 & & \\
After & 0.041 & & \\
\cline { 2 - 5 } Difference (After-Before) & -0.009 & 0.01 & 0.225 \\
\hline Difference-in-Differences & -0.051 & 0.01 & $<.001$ \\
\cline { 2 - 5 } & & & \\
\cline { 2 - 5 } & & &
\end{tabular}

Platelets units per bed day

Haematology

\begin{tabular}{r|l|l|l|l|}
\cline { 2 - 4 } Before & 0.161 & & \\
\cline { 2 - 5 } After & 0.145 & & \\
\hline Difference (After-Before) & -0.016 & 0.01 & 0.046 \\
\hline
\end{tabular}

The rest of $\mathrm{OUH}$

\begin{tabular}{r|l|l|l|} 
Before & 0.007 & & \\
After & 0.006 & & \\
\hline Difference (After-Before) & -0.001 & 0.01 & 0.896 \\
\hline Difference-in-Differences & -0.015 & 0.01 & 0.186 \\
\cline { 2 - 5 } & & &
\end{tabular}




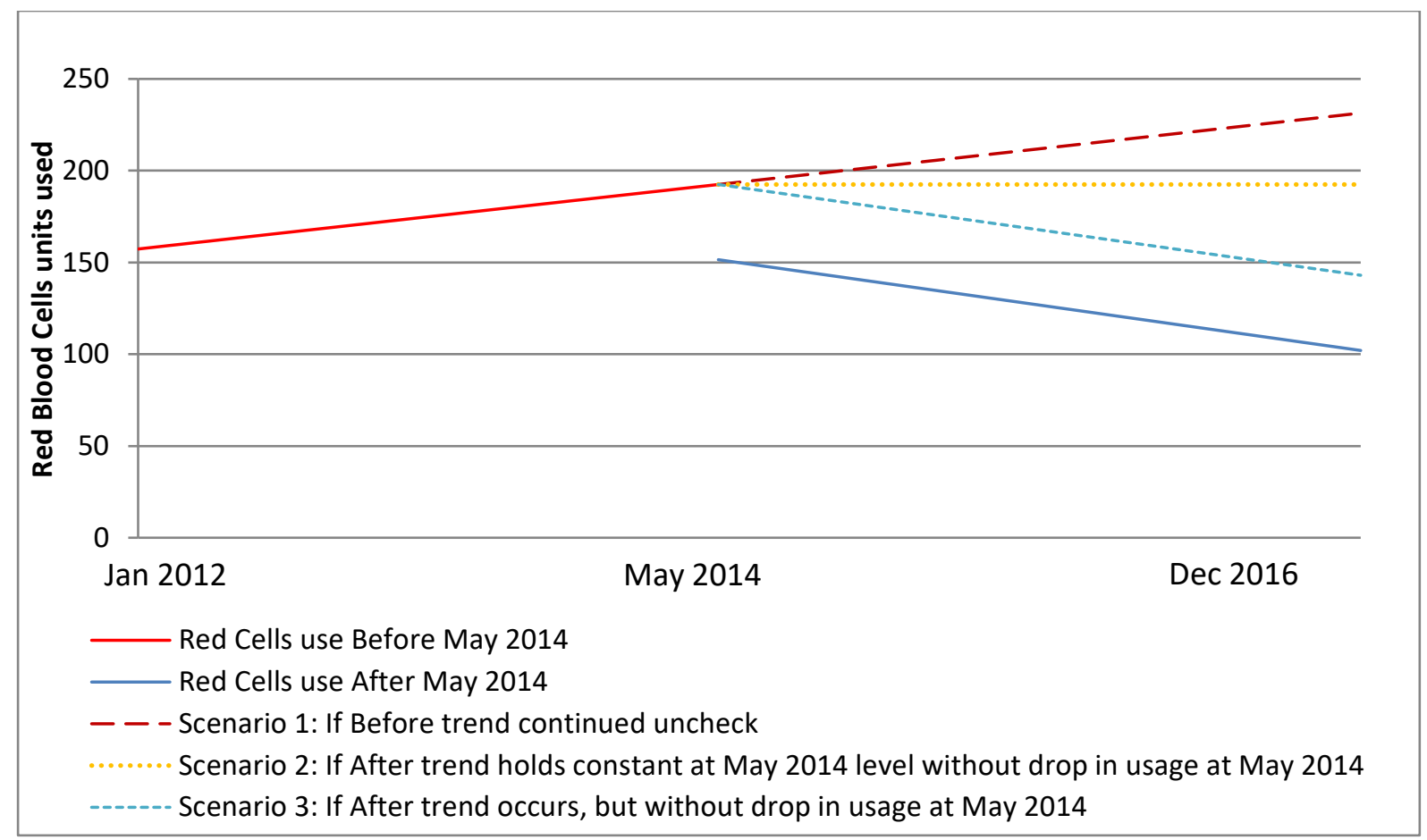

Figure 1: Red blood cells usage (piecewise regression and counter-factual scenarios) for the haematology department 


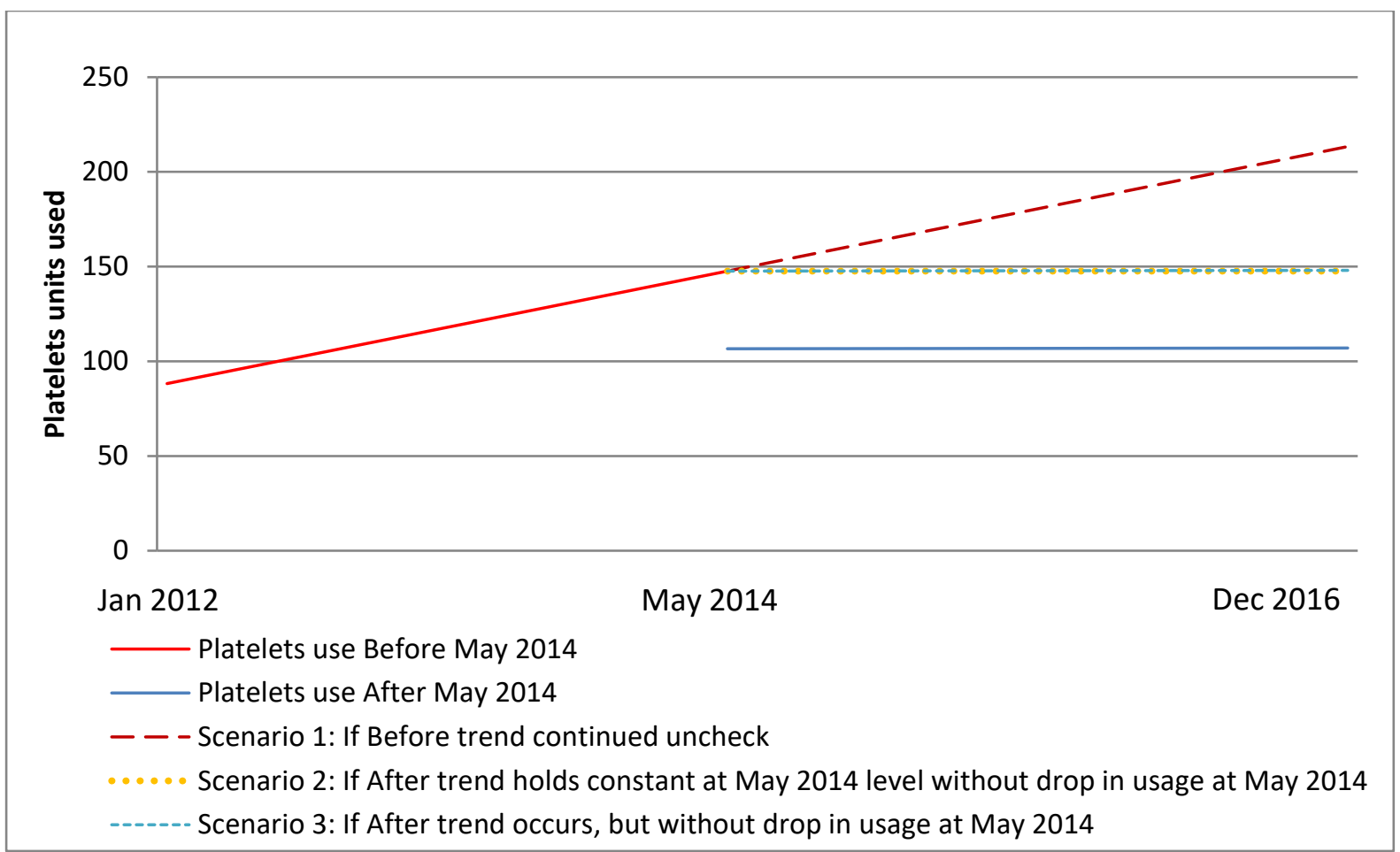

Figure 2: Platelets Usage (piecewise regression and counter-factual scenarios) for the haematology department. $\mathrm{Nb}$. In this figure, the lines for Scenarios 2 and 3 overlap, owing to the scale of the graph). 


\section{Economic Value of clinical decision support allied to direct data feedback to clinicians: blood usage in haematology - Supporting information}

\section{Supplementary material 1}

Supplementary Figure 1: raw data and regression trend lines for Red Blood Cells usage (haematology)

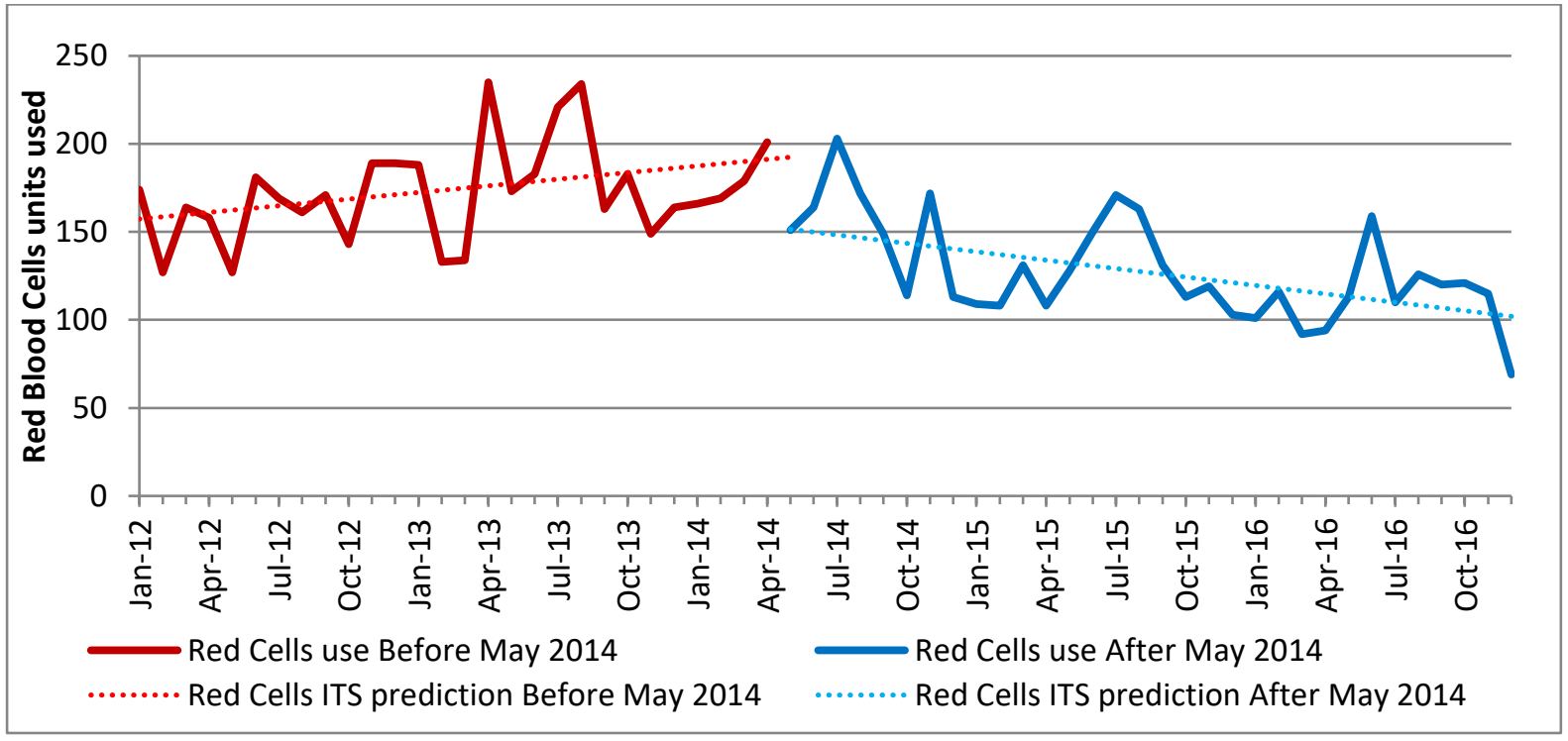

Supplementary Figure 2: raw data and regression trend lines for Platelets usage (haematology)

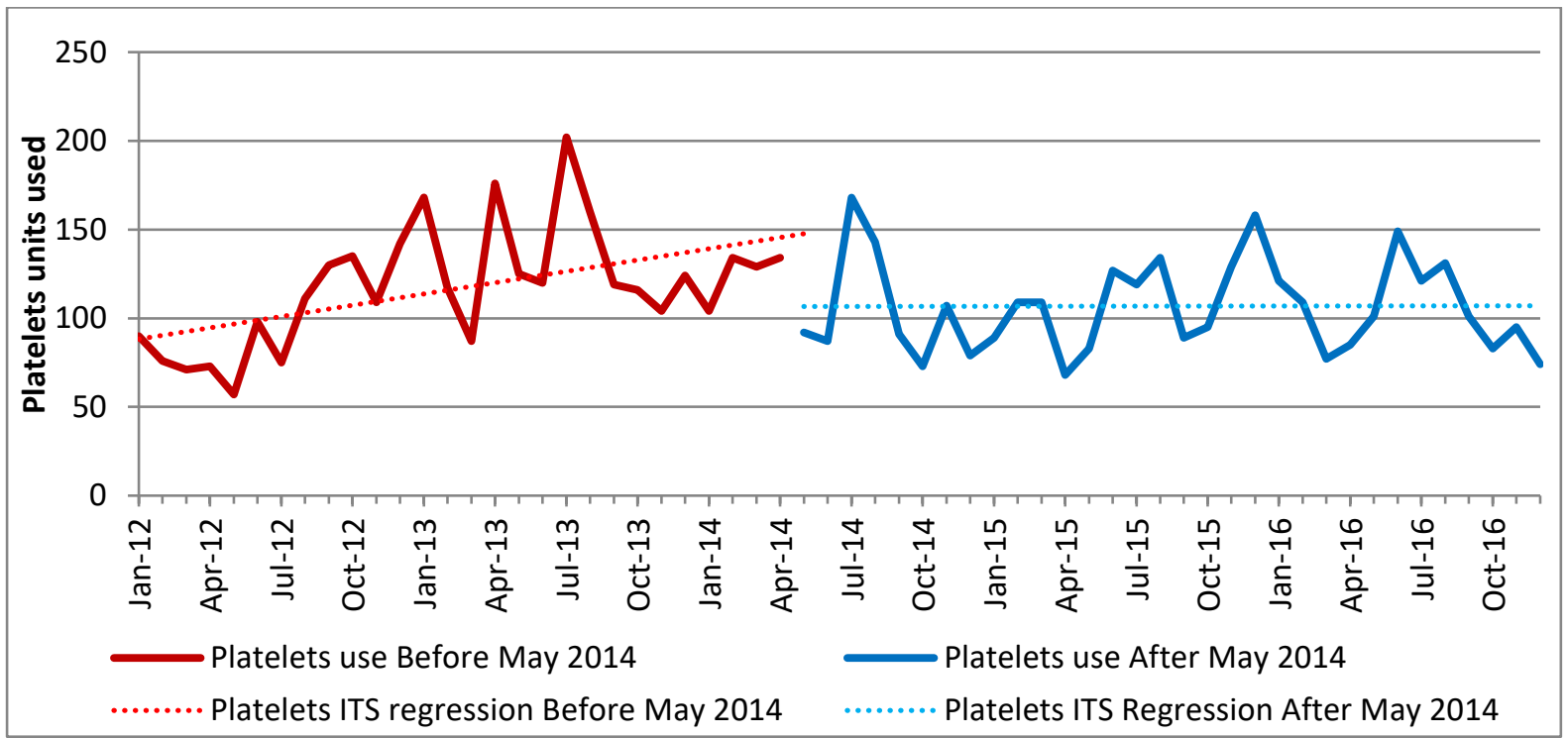




\section{Supplementary material 2}

Supplementary Figure 3: raw data and regression trend lines for Red Blood Cells usage (The rest of OUH)

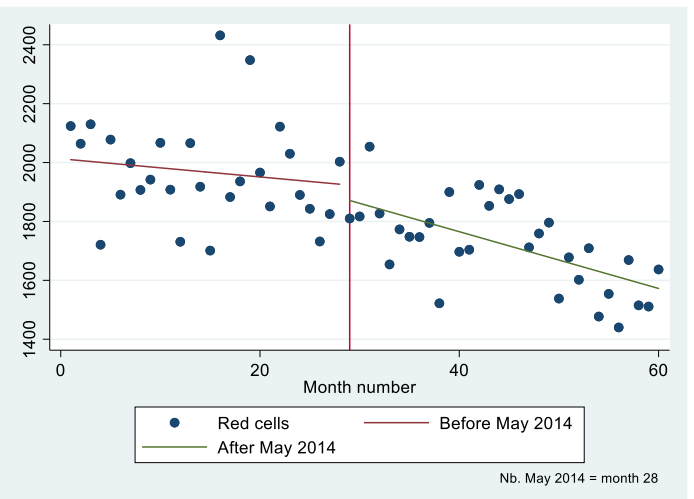

A similar trend to that of haematology can be observed in the rest of $\mathrm{OUH}$, although this trend is not as pronounced in the After period as the trend in haematology. In order to perform the Differencein-Differences analysis we needed to split the data into Before and After as with haematology and allow for a level-change at May 2014, so as to compare like with like. The observed drop in RBC and platelets usage in these graphs in therefore an artefact of the DiD regression, rather than a result of the intervention in haematology. 
Supplementary Figure 4: raw data and regression trend lines for Red Blood Cells usage (The rest of OUH)

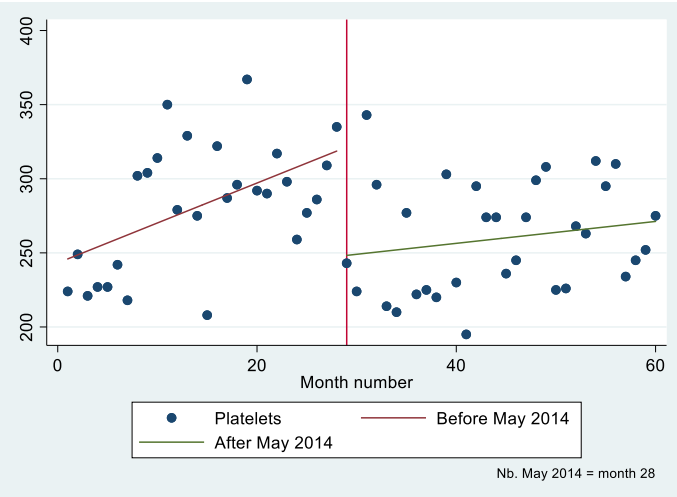

As stated for the RBC graph, the observed drop in platelets usage here is an artefact of the regression analysis that was necessary in order to perform an unbiased Difference-in-Difference analysis. The level-change is a much more apparent drop in usage than for RBC because of a much weaker correlation. 


\section{Supplementary material 3}

\section{Interrupted Time Series Model}

This ITS analysis used the following three variables:

- $\quad T$ is the time elapsed since the start of the study (in months)

- $X_{t}$ is a dummy variable indicating the pre-intervention period (coded 0 ) or the postintervention period (coded 1)

- $Y_{t}$ is the outcome at time $t$.

The equation of the regression model may be given as:

$$
Y_{t}=\beta_{0}+\beta_{1} T+\beta_{2} X_{t}+\beta_{3} T X_{t}
$$

Where $\beta_{0}$ represents the baseline level at $T=0, \beta_{1}$ is interpreted as the change in outcome associated with a 1-month increase (representing the underlying pre-intervention trend), $\beta_{2}$ is the level change following the intervention and $\beta_{3}$ indicates the slope change following the intervention (using the interaction between time and intervention: $T X_{t}$ ).

A seasonal effect on blood usage was observed in haematology for RBC (this effect was not present in platelets usage or in the rest of OUH's blood usage). As such we controlled for seasonality by including additional covariates (see below).

\section{Differences-in-Differences Model}

The equation for the DiD analysis may be given as:

$$
Z_{i t}=\beta_{0}+\beta_{1} X+\beta_{2} S+\beta_{3}(X \cdot S)+\epsilon_{i t}
$$

Where $Z_{i t}$ is the outcome at time $\mathrm{t}$ for unit $\mathrm{i}$, given by:

$$
\text { - } Z_{i t}=\frac{\alpha_{i t}}{\gamma_{i t}}
$$


- $\alpha_{i t}$ is the monthly blood product usage for haematology and the rest of OUH.

- $\gamma_{i t}$ is the number of bed days each month for haematology and the rest of OUH.

- $X$ is a dummy variable indicating the pre-intervention period (coded 0 ) or the postintervention period (coded 1 )

- $S$ is a dummy variable for haematology (coded 0$)$ or the rest of the OUH (coded 1$)$

- $\epsilon_{i t}$ is an error term

$Z$ is therefore the number of blood products used each month per bed day for haematology and the rest of OUH. 'Blood products' refers to RBC and platelets separately for individual DiD analyses.

These models were used to predict the trends in average monthly use of RBC and of platelets for haematology. The corresponding 'Before' and 'After' values for May 2014 and the slope of each trend line was then used to estimate the total volume of blood products used in each period. We repeated this analysis for the rest of OUH (excluding haematology).

\section{Seasonality in RBC in Haematology}

During our analysis we identified a problem with a seasonal pattern of usage in haematology, but only for RBC. This was observed in relatively high use of RBC in summer months, and relatively low use in winter months (see Figure below). Such autocorrelation violates the assumption of standard regression models that observations are independent. As such we controlled for seasonality by transforming the month variable into angles on the radians scale, such that January $=0$ and December $=11 / 12 * 2 \pi$.[26] We then included in the model a term for the sine and for the cosine of this transformed month variable. This means that we included $\beta_{\sin }$ and $\beta_{\cos }$ in the regression model for RBC in haematology, with the cosine function adjusted to have its maximum and minimum values in summer and winter, and the sine function for spring and autumn. The coefficients for the sine (transformed month) output from the regression model was -3.01 (95\% $\mathrm{Cl}-11.21$ to 5.19$)$ and for the cosine (transformed month) output was -18.8 ( $95 \% \mathrm{Cl}-27.00$ to 10.61). The cosine parameter 
was a significant predictor of RBC use (significant at $\mathrm{p}<0.05$ ). Controlling for this seasonality in this way removed the residual autocorrelation. This also considerably improved the regression model for haematology (improving the adjusted $\mathrm{R}^{2}$ value by 0.13 ). We could find no obvious explanation for the observed seasonality of RBC in haematology, however, the outcome of the analysis was not greatly influenced by controlling for seasonality except to improve the regression model.

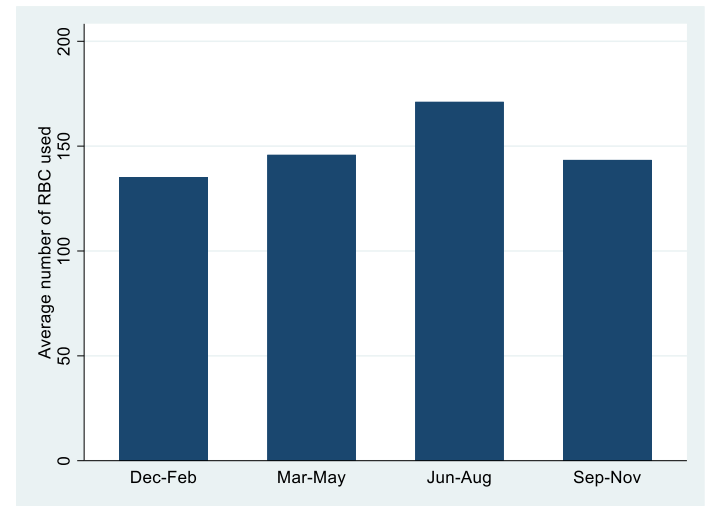

